TRABAJOS ORIGINALES

Rev Obstet Ginecol Venez. 2020; 80 (4):312-321

https://doi.org/10.51288/00800408

\title{
Evaluación endometrial vía histeroscopia en pacientes con cáncer de mama - receptores hormonales positivos, tratadas con hormonoterapia*
}

\author{
Drs. Carlos Lugo León, ${ }^{1}$ Nazira Monsalve. ${ }^{2}$
}

\section{RESUMEN}

Objetivo: Describir vía histeroscopia la patología endometrial asociada al uso de hormonoterapia en pacientes con cáncer de mama.

Métodos: El diseño de la investigación: no experimental, longitudinal, de tendencia; basado en pacientes que acudieron aleatoriamente, de enero a julio de 2014, a la consulta del Hospital Universitario de los Andes.

Resultados: La edad media de las pacientes fue de 55,3 años, tiempo medio de menopausia 8,03 años; 8 (26,6 \%) pacientes tenían asociado hipertensión arterial crónica y diabetes mellitus II y $10 \%$ de las pacientes cursaban con hipertensión arterial. Las alteraciones endometriales más comunes fueron: 46,7\% pólipos endometriales, 13,3\% hiperplasia simple sin atipias, 6,6\% leiomiomas. Entre las 5 (16,6\%) pacientes usuarias de exemestano, todas con ecografia normal, 3 (10\%) presentaron pólipos endometriales y 2 (6,7\%) biopsias negativas. Entre las 23 $(76,7 \%)$ pacientes que tomaban tamoxifeno, 9 (30\%) tenían endometrio engrosado, 12 (40\%) ecografia normal y $2(6,7 \%)$ otros hallazgos; 10 (33,3\%) presentaron pólipos (6 con ecografía normal y 4 con engrosamiento endometrial) y 3 hiperplasia endometrial (todas con endometrio engrosado). Dos pacientes que recibieron ambos tratamientos, una presentó hiperplasia y otra presentó pólipos (ambas con endometrio engrosado). La población estudiada en un $60 \%$ tenía cáncer de mama luminal B. Una paciente (3,3\%), tuvo un reflejo vagal como complicación del procedimiento.

Conclusión: Los hallazgos histeroscópicos con mayor frecuencia diagnosticados fueron los pólipos endometriales, hiperplasia simple sin atipias y miomas submucosos.

Palabras claves: Histeroscopia, endometrio, tamoxifeno, cáncer, mama.

\section{SUMMARY}

Objective: To know via hysteroscopy the endometrial pathology associated with the use of hormone therapy in patients with breast cancer.

Methods: Research design: non-experimental, longitudinal, trend; based on patients who come randomly, from January to July 2014, to the consultation of Los Andes University Hospital.

Results: The mean age of the patients was 55.3 years, mean time of menopause 8.03 years; 8 (26.6\%) patients had associated chronic arterial hypertension and diabetes mellitus II, and 10\% of the patients had arterial hypertension. The most common endometrial alterations were: $46.7 \%$ endometrial polyps, $13.3 \%$ simple hyperplasia without atypia, $6.6 \%$ leiomyomas. Among 5 (16.6\%) users of exemestane (all with normal ultrasound), 3 (10\%) had endometrial polyps and 2 (6.7\%) negative biopsies. Among the 23 (76.7\%) patients taking tamoxifen, 9 (30\%) had thickened endometrium, 12 (40\%) normal ultrasound and 2 (6.7\%) other findings; 10 (33.3\%) had polyps (6 with normal ultrasound and 4 with endometrial thickening) and 3 endometrial hyperplasia (all with thickened endometrium). Two patients receiving both treatments, one had hyperplasia and one had polyps (both with thickened endometrium). The population studies in 60\% were luminal B breast cancer; 1 (3.3\%) patient had a vagal reflex as a complication of the procedure.

Conclusion: Histeroscopic findings most often diagnosed were endometrial polyps, simple hyperplasia without atypia and submucosal myomas.

Keywords: Hysteroscopy, endometrium, tamoxifen, cancer, breast.

${ }^{1}$ Vicepresidente Junta Electoral Permanente, Miembro titular SOGV. Especialista en medicina materno fetal. Obstetra ginecólogo. ${ }^{2}$ Presidente Seccional Suroccidental, Miembro titular SOGV. Coordinadora postgrado obstetricia y ginecología, Universidad de los Andes. Obstetra ginecólogo.

*Trabajo realizado para optar el título de especialista en Obstetricia y Ginecología de la Universidad de los Andes.

\section{INTRODUCCIÓN}

La evaluación endometrial en toda paciente que acude a la consulta de ginecología es fundamental, especial atención se debe tener en las posmenopáusicas con 


\section{EVALUACIÓN ENDOMETRIAL VÍA HISTEROSCOPIA EN PACIENTES CON CÁNCER DE MAMA- RECEPTORES HORMONALES POSITIVOS, TRATADAS CON HORMONOTERAPIA}

factores de riesgo y/o comorbilidades asociadas para desarrollar alteraciones endometriales que ameriten manejo oportuno y eficaz. Tal es el caso de aquellas pacientes con cáncer de mama quienes reciben hormonoterapia, debido al aumento exponencial del riesgo para el desarrollo de cáncer de endometrio. La histeroscopia es la técnica de exploración directa que permite un fácil acceso visual al interior del cuello uterino y del útero para observar y evaluar el revestimiento de estas estructuras a través de un telescopio largo y delgado conectado a una fuente de luz llamado histeroscopio que se inserta a través de la vagina (1). Se puede utilizar para fines diagnósticos con mucha exactitud y elevada frecuencia, con fines terapéuticos y, en un alto porcentaje, de forma quirúrgica como: extracción de pólipos, liberación de sinequias, biopsias dirigidas, ablación endometrial, resección de miomas, entre otras (1). La técnica y el instrumental han experimentado una significativa evolución histórica a lo largo del tiempo hasta simplificarla y definirla como histeroscopia de quinta generación, un procedimiento sencillo, que no requiere anestesia, sedación, espéculo ni dilatación cervical. Sin embargo, la histeroscopia terapéutica para procedimientos invasivos se puede realizar con anestesia local, regional o general en quirófano (1).

El desarrollo de la histeroscopia tiene sus raíces en el trabajo de Pantaleoni (2), quien informó por primera vez sus estudios en 1869. Sin embargo, en ese momento la instrumentación era elemental y la expansión de la cavidad uterina era insuficiente (3). En 1925, Rubin utilizó por primera vez $\mathrm{CO}_{2}$ para distender el útero mientras que Gauss estaba experimentando con el uso de líquidos para lograr la expansión uterina (4). En Venezuela, los primeros reportes de histeroscopia se hicieron en el área de obstetricia y datan de 1966, según los reportes de Agüero y cols. (5) en Caracas y Arcay Solá (6) en la ciudad de Valencia.

En 1997, el Dr. Stefano Bettocchi introdujo sus diseños de quinta generación, conocido como el set de Bettocchi, el cual está basado en instrumentos ovalados de diámetro menor a $5 \mathrm{~mm}$ en una sola camisa diagnóstica y operatoria, simplificando el procedimiento a nivel del consultorio, sin anestesia, espéculo ni dilatación cervical; incorporó la vaginohisteroscopia y eliminó, casi por completo, la incomodidad de la paciente, de tal forma que no solo permite realizar un diagnóstico más certero sino resolver patologías específicas al más alto nivel tecnológico con un solo procedimiento (710). La histeroscopia diagnóstica es, en la actualidad, el método más fiable para el diagnóstico del cáncer endometrial, tanto como técnica aislada, como asociada a biopsia dirigida, directa o indirectamente, por lo que alcanza una sensibilidad de aproximadamente $100 \%$ (11).

Para el año 2009, Parker y cols. (12) establecieron el estado de los receptores hormonales para estrógeno (RE), progesterona (RP), HER2/neu y Ki-67, con lo cual el cáncer de mama es clasificado en luminal A (RE o RP positivos, HER2/neu negativo y bajo índice de proliferación), luminal B (RE o RP positivos, HER2/neu negativo e índice de proliferación alto) y luminal HER2/neu positivo (13). Los tipo luminal A y $\mathrm{B}$ son de buen pronóstico, pero el tipo $\mathrm{B}$ tiene un peor pronóstico que el tipo $\mathrm{A}$, debido a variaciones en la respuesta al tratamiento, pues expresan menor cantidad de RE; no obstante ambos se tratan con hormonoterapia (14).

El tratamiento hormonal en pacientes con cáncer de mama se basa principalmente en el tamoxifeno, el cual se utilizó por primera vez en 1960, en el Reino Unido, con efecto anticonceptivo (antiestrógeno trifenilenileno no esteroideo) (15). En 1966, debido al estudio de los distintos isómeros de la molécula, se descubre su efecto inductor de la ovulación (15). En 1977, la Food and Drug Administration (FDA) lo aprobó como tratamiento en el cáncer metastásico de mama en la posmenopausia y quince años más tarde quedaron demostrados sus beneficios en mujeres premenopáusicas así como beneficios asociados al 
intervalo libre de enfermedad y disminución del cáncer de mama contralateral en mujeres con tumores de mama RE positivos (15).

No es inusual la discordancia histeroscópicahistológica atribuible al edema de las glándulas y a la condensación del estroma periglandular inducidos por el tamoxifeno, el cual puede producir imágenes ecorrefringentes e irregulares de la línea endometrial al realizar exploraciones ecográficas (16). El riesgo de desarrollar cáncer de endometrio se estima en 1,7/1000 mujeres/año (17); este riesgo se incrementa 2 a 3 veces por el uso del tamoxifeno y estudios actuales refieren que mujeres con edades mayores a 35 años y tiempo de exposición al tratamiento mayor a 3 años, tienen más probabilidad de desarrollar carcinoma endometrial (OR 4,08. IC 95 \%: 1,67 -9,93) (18).

Los inhibidores no esteroideos de la aromatasa se subdividen de acuerdo al orden cronológico de su desarrollo clínico, siendo los de tercera generación (exemestano, letrozol y anastrozol) los más utilizados y aprobados para el tratamiento del cáncer de mama precoz en pacientes posmenopáusicas con receptores hormonales positivos (19). Ellos disminuyen los niveles de estrógenos inhibiendo la enzima aromatasa, responsable de la conversión de andrógenos a estrógenos. Estudios realizados han proporcionado pruebas que apoyan la administración de estos medicamentos en el tratamiento del cáncer de mama, ya sea por 5 años (terapia adyuvante) o después de 2,5 o 5 años de terapia adyuvante con base en tamoxifeno, como parte de una estrategia de conmutación (20).

Bissett y cols. (16) reportaron que la patología endometrial benigna más frecuente en estas pacientes son los pólipos $(60 \%)$ seguidos por las sinequias. El riesgo de neoplasia es mayor para tumores mesodérmicos mixtos y sarcomas que para el adenocarcinoma (ADC), células claras y carcinomas serosos papilares respectivamente (21).
En vista de que la histeroscopia es un método preciso para el diagnóstico de la patología endometrial porque proporciona una visión directa de la cavidad uterina, revela lesiones focales y permite biopsias dirigidas (22), se decidó realizar este estudio para evaluar la utilidad de la misma para establecer un diagnóstico y tratamiento oportuno, disminuir la incidencia de complicaciones y aumentar la sobrevida de las pacientes con cáncer de mama y hormonoterapia.

\section{MÉTODOS}

Se realizó un estudio observacional descriptivo para evaluar y documentar los cambios endometriales vía histeroscopia en pacientes con cáncer de mama y receptores hormonales positivos, con hormonoterapia, que acudieron a la consulta externa de Instituto Autónomo Hospital Universitario de los Andes en el periodo comprendido de enero a julio de 2014 . Los datos de las pacientes incluidos en el estudio se recogieron en un formato de recolección de datos ad hoc, que incluyó variables demográficas, clínicas y resultados de la evaluación histeroscópica así como los resultados histopatológicos.

A toda paciente que cumplió con los criterios de inclusión en el estudio y acudió a la consulta de patología mamaria, se le informó sobre el estudio (beneficios, técnica y posibles complicaciones), posterior a ello se le invitó a leer el consentimiento informado para que lo firmara y estampara su huella dactilar si estaba de acuerdo con participar en la investigación según lo expuesto.

Se procedió a realizar el estudio histeroscópico con el set de Bettocchi, marca Storz ${ }^{\circledR}$, con solución fisiológica $0,9 \%$ como medio de distensión, las posibles adherencias presentes en el canal endocervical así como en la cavidad endometrial fueron liberadas con tijera y/o grasping. 


\section{EVALUACIÓN ENDOMETRIAL VÍA HISTEROSCOPIA EN PACIENTES CON CÁNCER DE MAMA- RECEPTORES HORMONALES POSITIVOS, TRATADAS CON HORMONOTERAPIA}

Los hallazgos histeroscópicos observados durante el procedimiento fueron incluidos en la ficha de recolección de datos, asimismo la duración total del estudio y las posibles complicaciones durante el mismo. Se procedió posteriormente a correlacionar los hallazgos histeroscópicos con los obtenidos mediante estudios histológicos.

Se utilizó, para la recolección y procesamiento de los datos, el programa Microsoft Excel para Windows ${ }^{\circledR}$ año 2010.

\section{RESULTADOS}

En esta investigación se incluyeron 30 pacientes, 18 pacientes $(60 \%)$ cursaban con cáncer de mama luminal B y 12 (40\%) cáncer de mama luminal A. La edad media de las pacientes estudiadas fue de 55,3 $\pm 9,1$ años. En la tabla 1, se observa la distribución del diagnóstico histopatológico obtenido mediante histeroscopia: 18 pacientes con patología endometrial $(60 \%), 10 \sin$ lesiones $(33,3 \%)$ y dos con leiomiomas. Las lesiones tipo pólipos se presentaron en 14 pacientes $(46,7 \%), 7$ con 60 y más años $(23,3 \%)$, 4 pacientes entre los 50 - 59 años (13,3\%), y 3 (10\%) en menores de 50 años. La hiperplasia simple sin atipias se observó en 4 pacientes (13,3\%), 2 $(6,7 \%)$ de ellos en pacientes entre los $40-49$ años.
En relación con las comorbilidades presentes y los hallazgos histopatológicos, se encontraron 5 pacientes hipertensas $(16,7 \%)$, de las cuales 1 (3,3\%) presentó una hiperplasia simple sin atipias, 2 $(6,7 \%)$ presentaron pólipos endometriales y $2(6,7 \%)$ tuvieron hallazgos negativos. Una paciente diabética tuvo histeroscopia normal. Ocho pacientes $(26,6 \%)$ tenían ambas patologías: hipertensión arterial crónica (HTA) y diabetes mellitus tipo 2 (DM-2); entre ellas, $3(10 \%)$ presentaron hiperplasia simple sin atipias y $3(10 \%)$ pólipos endometriales. Dieciséis mujeres $(53,3 \%)$ no tenían ningún antecedente de importancia asociado, 9 pacientes (30 \%) sin comorbilidades presentaron pólipos endometriales, $5(16,7 \%)$ no tenían alteraciones y $2(6,7 \%)$ presentaron leiomiomas.

La menarquia comprendida entre los 9 - 12 años, sin comorbilidades asociadas, se observó en 10 pacientes $(33,3 \%)$, de las cuales 9 (30\%) tuvieron hallazgos anormales (miomas y pólipos endometriales). Seis pacientes $(20 \%)$ con alteraciones endometriales dadas por pólipos e hiperplasia simple sin atipias tenían asociado HTA y DM-2 y una menarquia más tardía (11 -13 años).

Entre las pacientes con menos de 4 años de menopausia, $10(33,3 \%)$ presentaron asociación a patología endometrial (hiperplasia simple sin atipias

Tabla 1. Distribución de diagnósticos histopatológicos obtenido por histeroscopia según los grupos etarios

\begin{tabular}{lccccc}
\hline & \multicolumn{5}{c}{$\begin{array}{c}\text { Diagnóstico histopatológico } \\
\mathrm{n}(\%)\end{array}$} \\
\cline { 2 - 6 } Edad & $\begin{array}{c}\text { Hiperplasia } \\
\text { simple sin atipias }\end{array}$ & Leiomioma & $\begin{array}{c}\text { Miomacoso/ } \\
\text { hipervascularización } \\
\text { endometrio }\end{array}$ & $\begin{array}{c}\text { Pólipo } \\
\text { endometrial }\end{array}$ & Negativo \\
\hline $30-39$ & - & - & - & $2(6,7)$ & - \\
$40-49$ & $2(6,7)$ & $1(3,3)$ & - & $1(3,3)$ & $3(10)$ \\
$50-59$ & $1(3,3)$ & - & $1(3,3)$ & $4(13,3)$ & $5(16,7)$ \\
60 y más & $1(3,3)$ & - & - & $7(23,3)$ & $2(6,7)$ \\
\hline
\end{tabular}


y pólipos endometriales) con un tiempo de exposición a hormonoterapia entre 2 y 4 años. Había 7 pacientes $(23,3 \%)$ con más de 16 años de menopausia, teniendo mayor asociación con pólipos endometriales. Las 4 pacientes con hiperplasia endometrial (13,3\%), tenían entre 1 y 4 años de menopausia, 1 había recibido tratamiento hormonal para la menopausia por menos de un año y 3 recibieron entre 2 y 3 años. De las 14 (46,7 \%) pacientes con pólipos, 4 (13,3\%) tenían entre 1 y 4 años de menopausia, todas, con menos de 4 años de tratamiento hormonal; 1 (3,3\%) tenía entre 5 y 8 años de menopausia y 2 años con tratamiento hormonal; dos pacientes $(6,7 \%)$ entre 9 y 12 años de menopausia, una $(3,3 \%)$ con menos de 1 año de tratamiento y otra $(3,3 \%)$ con 4 años de tratamiento; 7 pacientes $(23,3 \%)$ tenían más de 12 años de menopausia y todas recibieron tratamiento hormonal.

Al evaluar la asociación entre el uso de anticonceptivos orales (ACO) y los hallazgos histeroscópicos se observó que entre las 9 usuarias de ACO (30\%), 2 $(6,7 \%)$ tuvieron histeroscopia normal y 7 (23,3\%) pacientes presentaron hallazgos histeroscópicos anormales: $4(13,3 \%)$ tuvieron biopsias compatibles con pólipos y $1(3,3 \%)$ con hiperplasia endometrial. Entre las 21 no usuarias (70 \%), 18 (60\%) tuvieron histeroscopia con lesiones, hubo 3 (10\%) hiperplasias y $10(33,3 \%)$ pólipos (tabla 2$)$.
Tabla 2. Distribución de pacientes según el uso de anticonceptivos orales, los hallazgos histeroscópicos y el diagnóstico histopatológico

\begin{tabular}{lcc}
\hline & \multicolumn{1}{c}{$\begin{array}{c}\text { Hallazgo histeroscópico } \\
\text { anormal }\end{array}$} \\
$\begin{array}{l}\text { Hallazgos } \\
\text { histopatológicos }\end{array}$ & \multicolumn{2}{c}{$\mathrm{n}(\%)$} \\
\cline { 2 - 3 } & \multicolumn{2}{c}{$\begin{array}{c}\text { No usuaria } \\
\text { Usuaria }\end{array}$} \\
\hline $\begin{array}{l}\text { Hiperplasia simple sin } \\
\text { Leiomioma }\end{array}$ & $1(3,3)$ \\
$\begin{array}{l}\text { Mioma submucoso / Hiper- } \\
\text { vascularización endometrial }\end{array}$ & $1(3,3)$ & $1(3,3)$ \\
Negativo & $4(13,3)$ & $1(3,3)$ \\
Pólipo endometrial & $10(33,3)$ & $4(13,3)$ \\
\hline
\end{tabular}

Teniendo presente los diferentes tipos de tratamiento y los hallazgos histopatológicos obtenidos mediante histeroscopia se observa en la tabla 3 que entre las 5 $(16,6 \%)$ pacientes usuarias de solo exemestano, todas con ecografía normal, 3 (10\%) presentaron pólipos endometriales y $2(6,7 \%)$ biopsias negativas. Entre las $23(76,7 \%)$ pacientes que tomaban solo tamoxifeno, $9(30 \%)$ tenían endometrio engrosado, 12 (40\%) ecografía normal y $2(6,7 \%)$ otros hallazgos; 10 $(33,3 \%)$ presentaron pólipos (6 con ecografía normal y 4 con engrosamiento endometrial) y 3 hiperplasia

Tabla 3. Distribución de pacientes según el tratamiento hormonal y los hallazgos histopatológicos obtenidos por histeroscopia

\begin{tabular}{lccccc}
\hline & \multicolumn{5}{c}{$\begin{array}{c}\text { Diagnóstico histopatológico } \\
\mathrm{n}(\%)\end{array}$} \\
\cline { 2 - 6 } Tratamiento & $\begin{array}{c}\text { Mioma submucoso/ } \\
\text { Himple sin atipias }\end{array}$ & Leiomioma & $\begin{array}{c}\text { Hipervascularización } \\
\text { del endometrio }\end{array}$ & Negativo & $\begin{array}{c}\text { Pólipo } \\
\text { endometrial }\end{array}$ \\
\hline Exemestano & --- & --- & --- & $2(6,7)$ & $3(10)$ \\
Exemestano/ & $1(3,3)$ & --- & --- & -- & $1(3,3)$ \\
Tamoxifeno & $3(10)$ & $1(3,3)$ & $1(3,3)$ & $8(26,7)$ & $10(33,3)$ \\
Tamoxifeno & & & & & \\
\hline
\end{tabular}


Tabla 4. Distribución de pacientes según el tiempo de exposición al tratamiento hormonal para el cáncer de mama y los hallazgos histopatológicos obtenidos por histeroscopia

\begin{tabular}{lccccc}
\hline \multirow{2}{*}{$\begin{array}{l}\text { Tiempo } \\
\text { tratamiento } \\
\text { (años) }\end{array}$} & Leiomioma & $\begin{array}{c}\text { Diagnóstico histopatológico } \\
\mathrm{n}(\%)\end{array}$ \\
\cline { 2 - 6 } & $\begin{array}{c}\text { Mioma submucoso/ } \\
\text { Himple sin atipiascularización } \\
\text { del endometrio }\end{array}$ & Negativo & $\begin{array}{c}\text { Pólipo } \\
\text { endometrial }\end{array}$ \\
\hline$<1$ & $1(3,3)$ & --- & $1(3,3)$ & $1(3,3)$ & $2(6,7)$ \\
1 & --- & $1(3,3)$ & --- & $3(10)$ & $5(16,7)$ \\
2 & $3(10)$ & --- & --- & $4(13,3)$ & $1(3,3)$ \\
3 & --- & --- & --- & $1(3,3)$ & $3(10)$ \\
4 & --- & --- & & $1(3,3)$ & $3(10)$ \\
\hline
\end{tabular}

endometrial (todas con endometrio engrosado). Hubo dos pacientes que recibieron ambos tratamientos, una presentó hiperplasia y otra presentó pólipos (ambas con endometrio engrosado).

En la tabla 4 se observan las 18 pacientes $(60 \%)$ que presentaron alteraciones endometriales según la duración del tratamiento para el cáncer de mama. Antes de los dos primeros años de tratamiento hubo 7 $(23,3 \%)$ pacientes con pólipos y $1(3,3 \%)$ con hiperplasia endometrial. Con 2 años de tratamiento hubo $3(10 \%)$ hiperplasias y 1 (3,3\%) pólipo. En pacientes con 3 o más años de tratamiento, hubo 6 (20\%) pólipos.

Los reportes de ultrasonidos previos en comparación con los hallazgos y resultados obtenidos por histeroscopia se presentan en la tabla 5. Once pacientes tenían reportado en sus estudios de imágenes engrosamiento endometrial, los resultados histopatológicos reportaron 4 (13,3\%) hiperplasias y $5(16,7 \%)$ pólipos. Dos $(6,7 \%)$ pacientes tenían diagnóstico ecográfico de miomatosis y la histeroscopia mostró leiomiomatosis. Finalmente, en $17(56,7 \%)$ pacientes con ecografías normales, se encontraron 9 (30\%) casos de pólipos (tabla 5).

Tabla 5. Distribución de pacientes según los hallazgos histeroscópicos y el diagnóstico por ultrasonido

\begin{tabular}{lccccc}
\hline & \multicolumn{5}{c}{$\begin{array}{c}\text { Resultado histopatológico } \\
\mathrm{n}(\%)\end{array}$} \\
\cline { 2 - 6 } $\begin{array}{l}\text { Ultrasonido } \\
\text { ginecológico }\end{array}$ & $\begin{array}{c}\text { Hiperplasia } \\
\text { simple sin atipias }\end{array}$ & Leiomioma & $\begin{array}{c}\text { Mioma submucoso / } \\
\text { Hipervascularización } \\
\text { endometrio }\end{array}$ & $\begin{array}{c}\text { Pólipo } \\
\text { endometrial }\end{array}$ & Negativo \\
\hline $\begin{array}{l}\text { Engrosamiento } \\
\text { endometrial }\end{array}$ & $4(13,3)$ & -- & --- & $5(16,7)$ & $2(6,7)$ \\
$\begin{array}{l}\text { Miomatosis } \\
\text { uterina }\end{array}$ & --- & $1(3,3)$ & $1(3,3)$ & --- & --- \\
Sin alteraciones & --- & --- & --- & $9(30)$ & $8(26,7)$ \\
\hline
\end{tabular}




\section{DISCUSIÓN}

Las alteraciones endometriales en pacientes con cáncer de mama con receptores hormonales positivos tratadas con hormonoterapia están descritas en la literatura como uno de los efectos adversos más comunes que requieren seguimiento exhaustivo con el fin de descartar y tratar precozmente las diferentes patologías asociadas a su uso, evitando complicaciones o progresión de las mismas a patologías malignas.

Dentro de los diferentes métodos existentes para la evaluación endometrial, la histeroscopia se posiciona como el gold standard debido a la alta tasa de sensibilidad y especificidad que posee, ya que ofrece visión directa de la cavidad endometrial permitiendo el diagnóstico y tratamiento de las lesiones existentes al momento de su realización.

Teniendo presente el Comité de opinión del American College of Obstetricians and Gynecologists (ACOG), 2014 (23) donde recomiendan ampliar el uso de tamoxifeno a 10 años en vista de los beneficios adicionales que las pacientes pueden obtener en relación al cáncer de mama, este estudio se basó en la realización de vaginohisteroscopia de consultorio a todas las pacientes menopáusicas con el diagnóstico de cáncer de mama con receptores hormonales positivos quienes recibían tratamiento continuo con hormonoterapia, con la finalidad de evaluar los cambios endometriales asociados.

La edad media de las pacientes estudiadas fue de 55,3 años $\pm 9,1$ años. A pesar de que 53,3 \% de las pacientes no refirieron ningún tipo de comorbilidades, 33,3\% presentaron pólipos endometriales. Se observó que $20 \%$ de las pacientes que cursaban con HTA y DM-2 tuvieron reportes de biopsia para hiperplasia simple sin atipias $(10 \%)$ y pólipo endometrial $(10 \%)$, lo cual se asemeja con el estudio de Dalbert y cols. (24) quienes reportaron $28,95 \%$ de asociación de factores mixtos a patología endometrial.
La patología endometrial más frecuente en este estudio fueron las lesiones tipo pólipos en $46,6 \%$ de las pacientes, con predominio en mujeres mayores de 60 años (23,3\%); esto se relaciona con estudios como el de Bissett y cols. (16) quienes informaron que los pólipos son los más frecuentes; Le Donne y cols. (25) refirieron aproximadamente 30,9\% de pólipos en pacientes consumidoras de tamoxifeno y Andía y cols. (26) señalaron un aumento significativo de la patología endometrial, dada por pólipos endometriales, con la terapia hormonal.

La DM-2 como comorbilidad aislada no influyó en el desarrollo de patología endometrial, todo lo contrario a la HTA que estuvo asociada en $10 \%$ de las pacientes con patología endometrial, lo cual tiene relación con los trabajos de Dalbert y cols. (24) quienes informaron que $9,21 \%$ de las pacientes cursaban con HTA y patología endometrial.

La edad de aparición de la menarquia, como antecedente personal de importancia también fue evaluada, encontrándose un promedio de 12,06 años $\pm 1,6$ años, lo que tiene relación con los estudios realizado por Gómez (27) quien refiere que la edad de menarquia en Venezuela es de 12 años. En pacientes con menarquia entre los 11 y 13 años se observó la mayor asociación a patología endometrial (30 \%).

El uso de ACO fue relacionado con los hallazgos histeroscópicos, observándose pólipos endometriales en el 13,3 \% de las pacientes que refirieron su uso. Los hallazgos anormales fueron, en su mayoría, pólipos endometriales; el $60 \%$ de las pacientes informaron no haber consumido ACO en el pasado, con asociación de hallazgos histeroscópicos anormales, de estas pacientes 46,6\% presentaron alteraciones en el estudio histopatológico predominando los pólipos endometriales (33,3 \%) seguidos por la hiperplasia endometrial simple sin atipias $(10 \%)$. Estos resultados llaman la atención ya que sobre la base de estos hallazgos se pudiera 


\section{EVALUACIÓN ENDOMETRIAL VÍA HISTEROSCOPIA EN PACIENTES CON CÁNCER DE MAMA- RECEPTORES HORMONALES POSITIVOS, TRATADAS CON HORMONOTERAPIA}

considerar que haber consumido anticonceptivos orales previos al diagnóstico de cáncer de mama pudiera influir como factor protector del endometrio.

El tiempo promedio de menopausia fue de 8,03 años $\pm 6,9$ años. El 53,3\% de las pacientes tenían entre 1 y 4 años de menopausia, de estas 33,3\% presentaron alteraciones endometriales, predominantemente hiperplasia simple sin atipias $(13,3 \%)$ y pólipo endometrial (13,3\%). La mayor frecuencia de hiperplasia simple sin atipias se observó con 2 a 3 años de tratamiento mientras que para los pólipos endometriales de 3 a 4 años lo que se corresponde con el estudio de Pérez y cols. (28) quienes concluyeron que los patrones histeroscópicos correspondientes a pólipos son observados después de los 3 años de exposición al tamoxifeno.

La mayor incidencia de alteraciones endometriales corroboradas histopatológicamente se observó en aquellas pacientes consumidoras de tamoxifeno, de las cuales 33,3\% cursaron con pólipo endometrial, $10 \%$ pacientes con hiperplasia simple sin atipias y $6,6 \%$ de ellas con miomas submucosos. El tratamiento con exemestano se asoció con pólipo endometrial en $10 \%$ de las pacientes, mientras que tratamientos combinados (tamoxifeno/exemestano y exemestano/tamoxifeno) estaban relacionados con pólipo endometrial (3,3\%) e hiperplasia simple sin atipias $(3,3 \%)$ respectivamente.

En términos generales, la mayor frecuencia de lesiones asociadas al tiempo de exposición a la hormonoterapia se observó en aquellas pacientes con 1-2 años de tratamiento, con el $20 \%$ (16,6\% correspondiente a pólipos), seguidas de 13,3\% con tratamientos menores de 1 año y con $2-3$ años respectivamente.

Entre las 17 pacientes que presentaban estudios de ultrasonidos ginecológicos vía transvaginal sin alteraciones, se diagnosticó pólipo endometrial en el $30 \%$ de las pacientes, correspondiéndose con los estudios de Giorda y cols. (29) quienes reportaron que aproximadamente $38 \%$ de las pacientes tenían falsos negativos para patología endometrial vía ultrasonido ginecológico transvaginal.

El tiempo medio de duración del procedimiento fue de 8,4 minutos $\pm 2,69$ minutos, lo cual difiere del estudio de Gómez (27) quien reportó 15 min como duración media de la histeroscopia. En 3,3\% de las pacientes se observó una complicación inherente al procedimiento, dado por un síndrome vasovagal, el cual cedió posteriormente sin ningún tipo de tratamiento; por su parte, Pato y cols. (30) reportaron como complicación más frecuente el dolor intenso $(9,7 \%)$ y en segundo lugar el síndrome vagal (1\%).

En relación al dolor, todas las pacientes refirieron dolor leve, teniendo dichos hallazgos relación con el estudio de Bettocchi y cols. (31) quienes reportaron ausencia de dolor $\mathrm{y} / \mathrm{o}$ dolor leve durante el procedimiento; Sagiv y cols. (32) refirieron que las pacientes a las que se le administró anestesia intracervical percibían al final del estudio histeroscópico mayor dolor que aquellas pacientes que no recibieron anestesia lo cual no se correlaciona con estudios como el de Pato y cols. (30) quienes reportaron el dolor intenso en 9,7\% de sus pacientes.

La población de estudio estuvo conformada predominantemente por pacientes con cáncer de mama luminal $\mathrm{B}(60 \%)$, el resto presentaban cáncer de mama luminal A; esto no se relaciona con la literatura mundial, entre ellas la investigación de Imigo y cols. (13) quienes aseveraron que la mayor proporción de cáncer de mama es del tipo inmunohistoquímico luminal A.

Se concluye que el $60 \%$ de las pacientes estudiadas tenían diagnóstico previo de cáncer de mama luminal B. Los hallazgos histeroscópicos con mayor frecuencia diagnosticados fueron los pólipos endometriales (46,6\%), hiperplasia simple sin atipias (13,3\%), leiomiomas submucosos $(6,6 \%)$. La ingesta de 
tamoxifeno se asoció con una frecuencia de patología endometrial de $50 \%$, el exemestano se asoció en $10 \%$ y posteriormente los tratamientos combinados; el 43,3\% de las pacientes presentaron alteraciones endometriales en los primeros 3 años de tratamiento. La HTA asociada a DM-2 fueron las comorbilidades mayormente asociados a alteraciones endometriales. El uso de anticonceptivos orales no se asoció a mayor frecuencia en las alteraciones endometriales. Entre las 17 pacientes que presentaban estudios de ultrasonidos transvaginales sin alteraciones, $30 \%$ presentaron diagnóstico histeroscópico de pólipo endometrial. La duración promedio de realización de la histeroscopia de consultorio con toma de muestra endometrial fue de 8,4 $\pm 2,69$ minutos y solo se evidenció una complicación dada por reflejo vagal que mejoró con observación médica continua

\section{REFERENCIAS}

1. Chacón G. Complicaciones de la vagino-histeroscopia. $1^{\circ}$ taller de vagino-histeroscopia de consultorio. Sociedad de Obstetricia y Ginecología de Venezuela, seccional suroccidental. Mérida. 2012.

2. Pantaleoni DC. On endoscopic examination of the cavity of the womb. Med Press Circular. 1869; 8: 26 - 7.

3. Huertas M. Historia de la histeroscopia. Manual de histeroscopia diagnóstica y quirúrgica. Barcelona: Glosa; 2008.

4. Rubin IC. Uterine endoscopy, endometroscopy with the aid of uterine insuflation. Am J Obstet Gynecol. 1925; 10: 313 .

5. Aguero O, Aure M, López Mendoza R. Histeroscopia en embarazadas. Rev Obstet Ginecol Venez. 1967; 27 : $111-28$.

6. Arcay Solá A. Diagnóstico histeroscópico en el embarazo molar. Mayéutica 1967; 2:2.

7. Arias A. Histeroscopia diagnóstica en el sangrado postmenopáusico [Trabajo de ascenso]. Caracas (DC): Universidad Central de Venezuela; 1995.

8. Labastidas R, Arias A. Fiabilidad de la triada histerosalpingografía, histeroscopia, laparotomia. Memorias del Simposio Internacional Dexeus.
Reproducción Humana; 1981; Barcelona, España. 1981.

9. Van der Pas, H., Van Herendael, Bruno, Keith, L. Hysteroscopy. 1a ed. Dordrecht: Springer Nature Switzerland AG; 1983.

10. Arias A, Silva C. Removal of a knife piece by histeroscopy: A report of one case. Memories World Congress of Gynecologic Endoscopy; 18 al 22 de junio de 1997; Roma, Italia.

11. Coloma F, Costa S, Rodenas J, Payá V, Aliaga R, Reynal E, et al. Histeroscopia y cáncer: respuesta a las cuestiones planteadas tras dos décadas de experiencia. Prog Obstet Ginecol. 2010; 53 (9): 341 - 6. doi: 10.1016/j.pog.2010.04.003.

12. Parker J, Mullins M, Cheang M, Leung S, Voduc D, Vickery T, et al. Supervised Risk Predictor of Breast Cancer Based on Intrinsic Subtypes. J Clin Oncol. 2009; 27 (8): 1160 - 7. doi/10.1200/JCO.2008.18.1370.

13. Imigo F, Mansilla E, Delama I, Poblete M, Fonfach C. Clasificación molecular del cáncer de mama. Cuad Cir. 2011; 25 (1): 67 - 74. doi:10.4206/cuad.cir.2011. v25n1-10.

14. Sorlie T, Tibshirani R, Parker J, Hastie T, Marron JS, Nobel A, et al. Repeated observation of breast tumor subtypes in independent gene expression data sets. Proc Natl Acad Sci U S A 2003; 100 (14): 8418 - 23. doi: 10.1073/pnas.0932692100.

15. Mourits M, De Vires E, Willemse P, Ten Hoor K, Hollema H, Van der Zee A. Tamoxifen treatment and gynecologic side effects: A review. Obstet Gynecol. 2001; 97 (5): 855 - 66. doi: 10.1016/s00297844(00)01196-0.

16. Bissett D, Davis JA, George WD. Gynaecological monitoring during tamoxifen therapy. Lancet. 1994; 344 (8932): 1244. doi: 10.1016/s0140-6736(94)907471.

17. Bermejo J, Iniesta S, Redondo T, Gallego I, GarciaAndrade M. Cáncer uterino en pacientes tratadas con tamoxifeno por un cáncer de mama. Clin Invest Gin Obst. 2005; 32 (5): $204-8$.

18. Chen J, Kuo S, Liaw Y, Avital I, Stojadinovic A, Man $\mathrm{Y}$, et al. Endometrial cancer incidence in breast cancer patients correlating with age and duration of tamoxifen use: a population based study. J Cancer. 2014; 5 (2): 151 - 5. doi: 10.7150/jca.8412.

19. Van Asten K, Neven P, Lintermans A, Wildiers H, Paridaens R. Aromatase inhibitors in clinical breast 


\section{EVALUACIÓN ENDOMETRIAL VÍA HISTEROSCOPIA EN PACIENTES CON CÁNCER DE MAMA- RECEPTORES HORMONALES POSITIVOS, TRATADAS CON HORMONOTERAPIA}

cancer: focus on exemestane. Endocr Relat Cancer. 2014; 21(1): R31 - 49. doi: 10.1530/ERC-13-0269.

20. Gorostidi.M, Rivero B, Ecenarro E, Barinagarrementeria L, García M. Controversias en la prevención de patología endometrial en mujeres en tratamiento con tamoxifeno. Prog Obstet Ginecol. 2005; 48 (11): 521 8. doi: 10.1016/S0304-5013(05)72448-1.

21. Fisher B, Costantino JP, Redmond CK, Fisher ER, Wickerham DL, Cronin WM. Endometrial cancer in tamoxifen-treated breast cancer patients: findings from the National Surgical Adjuvant Breast and Bowel Project (NSABP) B-14. J Natl Cancer Inst. 1994; 86 (7): 527 - 37. doi: 10.1093/jnci/86.7.527.

22. Kalampokas T, Sofoudis C, Anastasopoulos C, Boutas I, Melloy S, Kondi-Pafiti A, et al. Effect of tamoxifeno on postmenopausal endometrium. Eur J Gynaecol Oncol 2013; 34 (4): $325-8$.

23. Committee opinion No. 601: Tamoxifen and Uterine Cancer. Obstet Gynecol. 2014; 123 (6): 1394 - 7. doi: 10.1097/01.AOG.0000450757.

24. Dalbert D, Rodriguez M, Figueredo A, Mural J, Bartt O, Subiela R, et al. Tamoxifeno y afecciones endometriales en pacientes con cáncer de mama. Medicina (Buenos Aires) 2013; 73 (2): 97 - 103.

25. Le Donne M, Alibrandi A, Ciancimino L, Azzerboni A, Chiófalo B, Triolo O. Endometrial Pathology in breast cancer patients: Effect of different treatments on ultrasonographic, histeroscopic and histological findings. Oncol. Lett. 2013; 5 (4): 1305 - 10. doi. org/10.3892/ol.2013.1156.

26. Andía D, Lafuente P, Matorras R, Usandizaga J. Efectos secundarios uterinos del tratamiento con tamoxifeno. Eur J Obstet Gynecol Reprod Biol . 2001; 1: 134 - 9.

27. Gómez H. Evaluación de la histeroscopia como método diagnóstico en las patologías endometriales [Trabajo Especial de Grado]. Mérida: Universidad de los Andes; 2011.
28. Pérez T, Salazar F, San-Frutos L, Ríos M, Jiménez J, Troyano J, et al. Hysteroscopic Dynamic Assessment of the Endometrium in Patients Treated with LongTerm Tamoxifen. J. Mimim. Invasive Gynecol. 2011; 18 (3): 349 - 54. doi: 10.1016/j.jmig.2010.12.014

29. Giorda G, Crivellari D, Veronesi A, Perin T, Campagnutta E, Carbone A, et al. Comparison of ultrasonography, hysteroscopy an biopsy in the diagnosis of endometrial lesions in postmenopausal tamoxifen-treated patients. Acta Obstet Gynecol Scand. 2002; 81 (10): 975 - 80. doi: 10.1034/j.1600-0412.2002.811013.x.

30. Pato M, Vásquez M, Pérez M, Garcia M, Blanco S. Indicaciones y resultados de la histeroscopia diagnóstica ambulatoria en el Complexo Hospitalario Universitario de Ourense. Ginecol Obstet Mex; 2013; 81 (07): 382 - 388.

31. Bettocchi S. Advanced Operative office Hysteroscopy without anesthesia: analysis of 501 cases treated with a 5 fr. Bipolar electrode. Hum Reprod. 2002; 17 (9): 2435 - 38. doi: 10.1093/humrep/17.9.2435.

32. Sagiv R, Sadan O, Boaz M, Dishi M, Schechter E, Golan A. A New Approach to Office Hysteroscopy Compared With Traditional Hysteroscopy. : a randomized controlled trial. Obstetrics \& Gynecology. 2006; 108 (2): 387 - 92. doi: 10.1097/01.AOG.0000227750.93984.0

Recibido: 20 de abril de 2020 Aprobado: 28 de junio de 2020 